\title{
Conditions for starlikeness of the Libera operator
}

Mamoru Nunokawa', Janusz Sokół², Nak Eun Cho ${ }^{3 *}$ and Oh Sang Kwon ${ }^{4}$

${ }^{\text {"Correspondence: }}$

necho@pknu.ac.kr

${ }^{3}$ Department of Applied

Mathematics, College of Natural

Sciences, Pukyong National

University, Pusan, 608-737, Korea

Full list of author information is

available at the end of the article

\begin{abstract}
Let $\mathcal{A}$ denote the class of functions $f$ that are analytic in the unit disc $\mathbb{D}$ and normalized by $f(0)=f^{\prime}(0)-1=0$. In this paper some conditions are determined for starlikeness of the Libera integral operator $F(z)=\frac{2}{z} \int_{0}^{z} f(t) \mathrm{d} t$.

MSC: Primary 30C45; secondary 30C80
\end{abstract}

Keywords: Nunokawa's lemma; starlike functions; strongly starlike functions

\section{Introduction}

Let $\mathcal{H}$ be the class of functions analytic in the unit disk $\mathbb{D}=\{z \in \mathbb{C}:|z|<1\}$, and let us denote by $\mathcal{A}_{n}$ the class of functions $f \in \mathcal{H}$ with the normalization of the form

$$
f(z)=z+a_{n+1} z^{n+1}+a_{n+2} z^{n+2}+\cdots, \quad z \in \mathbb{D},
$$

with $\mathcal{A}_{1}=\mathcal{A}$.

Let $\mathcal{S S}^{*}(\beta)$ denote the class of strongly starlike functions of order $\beta, 0<\beta \leq 1$,

$$
\mathcal{S S}^{*}(\beta)=\left\{f \in \mathcal{A}:\left|\arg \frac{z f^{\prime}(z)}{f(z)}\right|<\frac{\beta \pi}{2}, z \in \mathbb{D}\right\}
$$

which was introduced in [1] and [2], and $\mathcal{S S}^{*}(1) \equiv \mathcal{S}^{*}$ is the well-known class of starlike functions in $\mathbb{D}$. Functions in the class

$$
\mathcal{R}(\beta)=\left\{f \in \mathcal{A}: \mathfrak{R e}\left\{f^{\prime}(z)\right\}>\beta, z \in \mathbb{D}\right\},
$$

where $\beta<1$ are called functions with bounded turning. The Libera transform $L: \mathcal{A} \rightarrow \mathcal{A}$, $L[f]=F$, where

$$
F(z)=\frac{2}{z} \int_{0}^{z} f(t) \mathrm{d} t
$$

is the Libera integral operator, which has been studied by several authors on different counts. In [3] Mocanu considered the problem of starlikeness of $F$ and proved the following result.

Theorem 1.1 [3] Iff $(z)$ is analytic and $\mathfrak{R e}\left\{f^{\prime}(z)\right\}>0$ in the unit disc $\mathbb{D}$ and if the function $F$ is given in (1.1), then $F \in \mathcal{S}^{*}$.

\section{Springer}

○2014 Nunokawa et al.; licensee Springer. This is an Open Access article distributed under the terms of the Creative Commons Attribution License (http://creativecommons.org/licenses/by/2.0), which permits unrestricted use, distribution, and reproduction in any medium, provided the original work is properly cited. 
This result may be written briefly as follows:

$$
L[\mathcal{R}(0)] \subset \mathcal{S}^{*}=\mathcal{S S}^{*}(1)
$$

where $L[\mathcal{R}(0)]=\{L[f]: f \in \mathcal{R}(0)\}$. In 1995 Mocanu [4] improved (1.2) by showing that

$$
L[\mathcal{R}(0)] \subset \mathcal{S S}^{*}(8 / 9)
$$

In 2002 Miller and Mocanu [5] showed that a subcase of this last result can be sharpened to

$$
L\left[\mathcal{R}(0) \cap \mathcal{A}_{2}\right] \subset \mathcal{S S}^{*}(2 / 3)
$$

The problem of strongly starlikeness of $L[f]$ for $f \in \mathcal{R}(0)$ was consider also in [6] where it is shown that

$$
L\left[\mathcal{R}(0) \cap \mathcal{A}_{2}\right] \subset \mathcal{S S}^{*}(3 / 5)
$$

The above inclusion relationship is equivalent to the following differential implication:

$$
f \in \mathcal{A}_{2} \quad \text { and } \mathfrak{R e}\left\{f^{\prime}(z)\right\}>0 \Longrightarrow\left|\arg \left\{\frac{z F^{\prime}(z)}{F(z)}\right\}\right|<\frac{3 \pi}{10}
$$

or equivalently

$$
F \in \mathcal{A}_{2} \quad \text { and } \quad \mathfrak{R e}\left\{F^{\prime}(z)+\frac{1}{2} z F^{\prime \prime}(z)\right\}>0 \Rightarrow\left|\arg \left\{\frac{z F^{\prime}(z)}{F(z)}\right\}\right|<\frac{3 \pi}{10}
$$

where $F$ is given by (1.1).

In [7] Ponnusamy improved (1.2) by showing that

$$
L[\mathcal{R}(-\varrho)] \subset \mathcal{S}^{*}, \quad \varrho=0.09032572 \ldots
$$

On the order of starlikeness of convex functions was considered also in the recent paper [8].

\section{Main result}

In this paper we go back to the problem of starlikeness of Libera transform. We need the following lemmas.

Lemma 2.1 [9, p.73] Let $n$ be a positive integer, $\lambda>0$ and let $\beta_{0}=\beta_{0}(\lambda, n)$ be the positive root of the equation

$$
\beta \pi=3 \pi / 2-\tan ^{-1}(n \lambda \beta) .
$$

In addition, let

$$
\alpha=\alpha(\beta, \lambda, n)=\beta+(2 / \pi) \tan ^{-1}(n \lambda \beta)
$$


for $0<\beta \leq \beta_{0}$. If $p(z)=1+p_{n} z^{n}+p_{n+1} z^{n+1}+\cdots$ is analytic in $\mathbb{D}$, then

$$
p(z)+\lambda z p^{\prime}(z) \prec\left(\frac{1+z}{1-z}\right)^{\alpha}, \quad z \in \mathbb{D}
$$

implies the following subordination:

$$
p(z) \prec\left(\frac{1+z}{1-z}\right)^{\beta}, \quad z \in \mathbb{D} .
$$

If in Lemma 2.1 we put $n=1, \lambda=1 / 2$, then the solution $\beta_{0}$ of (2.1) satisfies $\beta_{0}>1$, so we may take $\beta=1$, which gives $\pi \alpha / 2=\pi / 2+\tan ^{-1}(1 / 2)=2.03 \ldots$

Corollary 2.2 Assume that $f(z) \in \mathcal{A}_{1}$. If

$$
\left|\arg \left\{F^{\prime}(z)+(1 / 2) z F^{\prime \prime}(z)\right\}\right|<\pi / 2+\tan ^{-1}(1 / 2)=2.03 \ldots, \quad z \in \mathbb{D}
$$

then

$$
\mathfrak{R e}\left\{F^{\prime}(z)\right\}>0, \quad z \in \mathbb{D} \text {. }
$$

Note that if $F(z) \in \mathcal{A}_{2}$, then a sufficient condition for $F \in \mathcal{R}(0)$ is $\left|\arg \left\{f^{\prime}(z)\right\}\right|<3 \pi / 4=$ $2.356 \ldots$; see $[5, \mathrm{p} .96]$.

Lemma 2.3 [10] Let $p(z)$ be of the form

$$
p(z)=1+\sum_{n=m \geq 1}^{\infty} a_{n} z^{n}, \quad a_{m} \neq 0(z \in \mathbb{D}),
$$

with $p(z) \neq 0$ in $\mathbb{D}$. If there exists a point $z_{0},\left|z_{0}\right|<1$, such that

$$
|\arg \{p(z)\}|<\pi \alpha / 2 \text { in }|z|<\left|z_{0}\right| \text { and } \quad\left|\arg \left\{p\left(z_{0}\right)\right\}\right|=\pi \alpha / 2
$$

for some $\alpha>0$, then we have

$$
\frac{z_{0} p^{\prime}\left(z_{0}\right)}{p\left(z_{0}\right)}=i k \alpha
$$

where

$$
k \geq m\left(a^{2}+1\right) /(2 a), \quad \text { when } \arg \left\{p\left(z_{0}\right)\right\}=\pi \alpha / 2
$$

and

$$
k \leq-m\left(a^{2}+1\right) /(2 a), \quad \text { when } \arg \left\{p\left(z_{0}\right)\right\}=-\pi \alpha / 2
$$

where

$$
\left\{p\left(z_{0}\right)\right\}^{1 / \alpha}= \pm i a, \quad a>0
$$


Lemma 2.4 [9, p.75], [11] Let $p(z)=1+\sum_{n=1}^{\infty} a_{n} z^{n}$ be analytic in the unit disc $\mathbb{D}$. If

$$
p(z)+z p^{\prime}(z) \prec \frac{1+z}{1-z}, \quad z \in \mathbb{D},
$$

then

$$
p(z) \prec q(z)=\frac{2}{z} \log \frac{1}{1-z}-1
$$

and

$$
|\arg \{p(z)\}|<\theta_{0}=\max _{|z|=1}|\arg \{q(z)\}|=0.9110 \ldots, \quad z \in \mathbb{D},
$$

where $\theta_{0}$ lies between 0.911621904 and 0.911621907 .

Theorem 2.5 Let $q(z)$ be analytic in $\mathbb{D}$ and suppose that

$$
|\arg \{q(z)\}|<\frac{\beta \pi}{2}, \quad z \in \mathbb{D}
$$

for some $\beta \in(0,1]$. If $p(z)$ is analytic and $p(z) \neq 0$ in $\mathbb{D}$ with $p(0)=1$ and such that

$$
\left|\arg \left\{q(z)\left(z p^{\prime}(z)+p^{2}(z)+p(z)\right)\right\}\right|<\tan ^{-1} \beta, \quad z \in \mathbb{D},
$$

then we have

$$
|\arg \{p(z)\}|<\frac{\beta \pi}{2}, \quad z \in \mathbb{D} .
$$

Proof If there exists a point $z_{0},\left|z_{0}\right|<1$, for which

$$
|\arg \{p(z)\}|<\pi \beta / 2 \quad\left(|z|<\left|z_{0}\right|\right)
$$

and

$$
\left|\arg \left\{p\left(z_{0}\right)\right\}\right|=\pi \beta / 2, \quad p\left(z_{0}\right)=( \pm i a)^{\beta},
$$

then from Nunokawa's Lemma 2.3, we have

$$
\frac{z_{0} p^{\prime}\left(z_{0}\right)}{p\left(z_{0}\right)}=i k \beta
$$

where

$$
k \geq \frac{a^{2}+1}{2 a} \geq 1, \quad \text { when } \arg \left\{p\left(z_{0}\right)\right\}=\pi \beta / 2
$$

and

$$
k \leq-\frac{a^{2}+1}{2 a} \leq-1, \quad \text { when } \arg \left\{p\left(z_{0}\right)\right\}=-\pi \beta / 2 .
$$


For the case $\arg \left\{p\left(z_{0}\right)\right\}=\beta \pi / 2$, we have

$$
\begin{aligned}
& \left|\arg \left\{q\left(z_{0}\right)\left[z_{0} p^{\prime}\left(z_{0}\right)+p^{2}\left(z_{0}\right)+p\left(z_{0}\right)\right]\right\}\right| \\
& \quad=\left|\arg \left\{q\left(z_{0}\right) p\left(z_{0}\right)\left[1+p\left(z_{0}\right)+z_{0} p^{\prime}\left(z_{0}\right) / p\left(z_{0}\right)\right]\right\}\right| \\
& \quad=\left|\frac{\pi \beta}{2}+\arg \left\{q\left(z_{0}\right)\right\}+\arg \left\{1+p\left(z_{0}\right)+z_{0} p^{\prime}\left(z_{0}\right) / p\left(z_{0}\right)\right\}\right| \\
& \quad=\left|\frac{\pi \beta}{2}+\arg \left\{q\left(z_{0}\right)\right\}+\tan ^{-1}\left\{\frac{\beta k+a^{\beta} \sin (\pi \beta / 2)}{1+a^{\beta} \cos (\pi \beta / 2)}\right\}\right|,
\end{aligned}
$$

where $p\left(z_{0}\right)=(i a)^{\beta}, 0<a$ and

$$
k \geq \frac{a^{2}+1}{2 a} \geq 1
$$

Let us put

$$
g(a)=\frac{k \beta+a^{\beta} \sin (\pi \beta / 2)}{1+a^{\beta} \cos (\pi \beta / 2)}, \quad 0<a,
$$

then it is easy to see that

$$
g(a) \geq \frac{\beta+a^{\beta} \sin (\pi \beta / 2)}{1+a^{\beta} \cos (\pi \beta / 2)}, \quad 0<a .
$$

Putting

$$
h(x)=\frac{\beta+x \sin (\pi \beta / 2)}{1+x \cos (\pi \beta / 2)}, \quad 0 \leq x
$$

we have

$$
h^{\prime}(x)=\frac{\sin (\pi \beta / 2)-\beta \cos (\pi \beta / 2)}{(1+x \cos (\pi \beta / 2))^{2}}>0, \quad 0 \leq x,
$$

because $\tan (\pi \beta / 2)>\beta$. Therefore, for $x>0$ we get $h(x)>h(0)=\beta$, so from (2.11) we have

$$
g(a)>\beta
$$

and so

$$
\tan ^{-1}\left\{\frac{k \beta+a^{\beta} \sin (\pi \beta / 2)}{1+a^{\beta} \cos (\pi \beta / 2)}\right\}>\tan ^{-1} \beta, \quad 0<a .
$$

Therefore, we have the following inequality from (2.10):

$$
\begin{aligned}
& \left|\arg \left\{q\left(z_{0}\right)\left[z_{0} p^{\prime}\left(z_{0}\right)+p^{2}\left(z_{0}\right)+p\left(z_{0}\right)\right]\right\}\right| \\
& \quad \geq \frac{\pi \beta}{2}+\tan ^{-1} \frac{k+a^{\beta} \sin (\pi \beta / 2)}{1+a^{\beta} \cos (\pi \beta / 2)}-\left|\arg \left\{q\left(z_{0}\right)\right\}\right| \\
& \quad>\tan ^{-1} \beta .
\end{aligned}
$$


This contradicts the hypothesis and for the case $\arg \left\{p\left(z_{0}\right)\right\}=-\beta \pi / 2$, applying the same method as above, we also have (2.12). This is also a contradiction and it completes the proof.

Corollary 2.6 Assume that

$$
\left|\arg \left\{f^{\prime}(z)\right\}\right|<\tan ^{-1} \beta, \quad z \in \mathbb{D}
$$

and

$$
|\arg \{F(z) / z\}|<\frac{\beta \pi}{2}, \quad z \in \mathbb{D}
$$

for some $\beta \in(0,1]$, where $F(z)$ is given in (1.1). Then we have

$$
\left|\arg \left\{\frac{z F^{\prime}(z)}{F(z)}\right\}\right|<\frac{\beta \pi}{2}, \quad z \in \mathbb{D}
$$

hence $F(z)$ is strongly starlike of order $\beta$.

Proof If we set

$$
p(z)=\frac{z F^{\prime}(z)}{F(z)}
$$

then

$$
f^{\prime}(z)=F^{\prime}(z)+\frac{1}{2} z F^{\prime \prime}(z)=\frac{1}{2}\left(\frac{F(z)}{z}\right)\left(z p^{\prime}(z)+p^{2}(z)+p(z)\right) .
$$

If we let $q(z)=F(z) / z$, then by (2.14) and (2.15) the assumptions of Theorem 2.5 are satisfied. Therefore,

$$
|\arg \{p(z)\}|<\frac{\beta \pi}{2}, \quad z \in \mathbb{D} .
$$

Theorem 2.7 Let $q(z)$ be analytic in $\mathbb{D}$, with $q(0)=1$ and satisfy

$$
\mathfrak{R e}\left\{z q^{\prime}(z)+q(z)\right\}>0, \quad z \in \mathbb{D} .
$$

If $p(z)$ is analytic in $\mathbb{D}$, with $p(0)=1$ and if

$$
\left|\arg \left\{q(z)\left(z p^{\prime}(z)+p^{2}(z)+p(z)\right)\right\}\right|<\frac{5 \pi}{6}-\theta_{0}=1.706 \ldots, \quad z \in \mathbb{D},
$$

where $\theta_{0}$ is given in (2.8), then we have

$$
\mathfrak{R e}\{p(z)\}>0, \quad z \in \mathbb{D} .
$$

Proof By Lemma 2.4, we have

$$
|\arg \{q(z)\}|<\theta_{0}=0.911 \ldots, \quad z \in \mathbb{D} .
$$


If there exists a point $z_{0},\left|z_{0}\right|<1$, such that

$$
|\arg \{p(z)\}|<\pi / 2 \quad\left(|z|<\left|z_{0}\right|\right)
$$

and

$$
\left|\arg \left\{p\left(z_{0}\right)\right\}\right|=\pi / 2, \quad p\left(z_{0}\right)= \pm i a, 0<a,
$$

then from Nunokawa's Lemma 2.3, we have

$$
\frac{z_{0} p^{\prime}\left(z_{0}\right)}{p\left(z_{0}\right)}=i k
$$

where

$$
k \geq \frac{a^{2}+1}{2 a} \geq 1, \quad \text { when } \arg \left\{p\left(z_{0}\right)\right\}=\pi / 2
$$

and

$$
k \leq-\frac{a^{2}+1}{2 a} \leq-1, \quad \text { when } \arg \left\{p\left(z_{0}\right)\right\}=-\pi / 2 .
$$

For the case $\arg \left\{p\left(z_{0}\right)\right\}=\pi / 2$, we have

$$
\begin{aligned}
\arg \{1+i a+i k\} & \geq \arg \left\{1+i a+i \frac{a^{2}+1}{2 a}\right\} \\
& =\tan ^{-1} \frac{\mathfrak{I m}\left\{1+i a+i \frac{a^{2}+1}{2 a}\right\}}{\mathfrak{R e}\left\{1+i a+i \frac{a^{2}+1}{2 a}\right\}} \\
& =\tan ^{-1}\left\{\frac{3 a^{2}+1}{2 a}\right\} \\
& \geq \tan ^{-1}\{\sqrt{3}\} \\
& =\frac{\pi}{3} .
\end{aligned}
$$

Therefore, for the case $\arg \left\{p\left(z_{0}\right)\right\}=\pi / 2$, we have

$$
\frac{\pi}{3} \leq \arg \{1+i a+i k\}<\frac{\pi}{2} .
$$

Moreover, by (2.16)

$$
\arg \left\{q\left(z_{0}\right)\right\}<\theta_{0} .
$$

Therefore, we can write

$$
\begin{aligned}
& \left|\arg \left\{q\left(z_{0}\right)\left(z_{0} p^{\prime}\left(z_{0}\right)+p^{2}\left(z_{0}\right)+p\left(z_{0}\right)\right)\right\}\right| \\
& \quad=\left|\arg \left\{p\left(z_{0}\right)\left[1+p\left(z_{0}\right)+z_{0} p^{\prime}\left(z_{0}\right) / p\left(z_{0}\right)\right] q\left(z_{0}\right)\right\}\right| \\
& \quad \geq\left|\arg \left\{p\left(z_{0}\right)(1+i a+i k)\right\}\right|-\left|\arg \left\{q\left(z_{0}\right)\right\}\right|
\end{aligned}
$$




$$
\begin{aligned}
& \geq \frac{\pi}{2}+\frac{\pi}{3}-\left|\arg \left\{q\left(z_{0}\right)\right\}\right| \\
& \geq \frac{5 \pi}{6}-\theta_{0} .
\end{aligned}
$$

This contradicts the hypothesis and for the case $\arg \left\{p\left(z_{0}\right)\right\}=-\pi / 2$, applying the same method as above, we have

$$
\left|\arg \left\{q\left(z_{0}\right)\left(z_{0} p^{\prime}\left(z_{0}\right)+p^{2}\left(z_{0}\right)+p\left(z_{0}\right)\right)\right\}\right| \geq \frac{5 \pi}{6}-\theta_{0} .
$$

This is also a contradiction and it completes the proof.

\section{Corollary 2.8 Assume that}

$$
\left|\arg \left\{f^{\prime}(z)\right\}\right|<\frac{5 \pi}{6}-\theta_{0}=1.706 \ldots, \quad z \in \mathbb{D},
$$

then we have

$$
\mathfrak{R e}\left\{\frac{z F^{\prime}(z)}{F(z)}\right\}>0, \quad z \in \mathbb{D}
$$

where $F(z)$ is Libera integral given in (1.1).

Proof Because

$$
f^{\prime}(z)=F^{\prime}(z)+\frac{1}{2} z F^{\prime \prime}(z)
$$

by Corollary 2.2 and by (2.18) we obtain

$$
\mathfrak{R e}\left\{F^{\prime}(z)\right\}>0, \quad z \in \mathbb{D} \text {. }
$$

Therefore, if we let $q(z)=F(z) / z$, then

$$
\mathfrak{R e}\left\{z q^{\prime}(z)+q(z)\right\}=\mathfrak{R e}\left\{F^{\prime}(z)\right\}>0, \quad z \in \mathbb{D} .
$$

If we set

$$
p(z)=\frac{z F^{\prime}(z)}{F(z)}
$$

then

$$
f^{\prime}(z)=F^{\prime}(z)+\frac{1}{2} z F^{\prime \prime}(z)=\frac{1}{2}\left(\frac{F(z)}{z}\right)\left(z p^{\prime}(z)+p^{2}(z)+p(z)\right) .
$$

The assumptions of Theorem 2.7 are satisfied. Therefore, (2.19) holds.

Corollary 2.8 is an extension of Mocanu's result (1.2) from the paper [3] because in (2.18) we have $\left|\arg \left\{f^{\prime}(z)\right\}\right|<1.706 \ldots$, while in (1.2) we have the stronger assumption that $\left|\arg \left\{f^{\prime}(z)\right\}\right|<\pi / 2=1.57 \ldots$ 


\section{Competing interests}

The authors declare that they have no competing interests.

\section{Authors' contributions}

All authors jointly worked on the results and they read and approved the final manuscript.

\section{Author details}

${ }^{1}$ University of Gunma, Hoshikuki-cho 798-8, Chuou-Ward, Chiba, 260-0808, Japan. ${ }^{2}$ Department of Mathematics, Rzeszów University of Technology, Al. Powstańców Warszawy 12, Rzeszów, 35-959, Poland. ${ }^{3}$ Department of Applied Mathematics, College of Natural Sciences, Pukyong National University, Pusan, 608-737, Korea. ${ }^{4}$ Department of Mathematics, Kyungsung University, Busan, 608-736, Korea.

\section{Acknowledgements}

The authors would like to express their thanks to the referees for valuable advice regarding a previous version of this paper. This research was supported by the Basic Science Research Program through the National Research Foundation of Korea (NRF) funded by the Ministry of Education, Science and Technology (No. 2011-0007037).

\section{Received: 31 December 2013 Accepted: 7 March 2014 Published: 31 Mar 2014}

\section{References}

1. Stankiewicz, J: Quelques problèmes extrèmaux dans les classes des fonctions $\alpha$-angulairement ètoilèes. Ann. Univ. Mariae Curie-Skłodowska, Sect. A 20, 59-75 (1966)

2. Brannan, DA, Kirwan, WE: On some classes of bounded univalent functions. J. Lond. Math. Soc. (2) 1, $431-443$ (1969)

3. Mocanu, PT: On starlikness of Libera transform. Mathematica 28(51), 153-155 (1986)

4. Mocanu, PT: New extensions of a theorem of R. Singh and S. Singh. Mathematica 37(60), 171-182 (1995)

5. Miller, SS, Mocanu, PT: Libera transform of functions with bounded turning. J. Math. Anal. Appl. 276, 90-97 (2002)

6. Sokół, J: Starlikeness of the Libera transform of functions with bounded turning. Appl. Math. Comput. 203, 273-276 (2008)

7. Ponnusamy, S: On starlikeness of certain integral transforms. Ann. Pol. Math. 40, 227-232 (1992)

8. Nunokawa, M, Sokół, J: On the order of strongly starlikeness of convex functions of order alpha. Mediterr. J. Math. (2013). doi:10.1007/s00009-013-0341-6

9. Miller, SS, Mocanu, PT: Differential Subordinations: Theory and Applications. Series of Monographs and Textbooks in Pure and Applied Mathematics, vol. 225. Dekker, New York (2000)

10. Nunokawa, M: On the order of strongly starlikeness of strongly convex functions. Proc. Jpn Acad., Ser. A 69, 234-237 (1993)

11. Mocanu, PT, Ripeanu, D, Popovici, M: The best bound for the argument of certain analytic functions with positive real part. Babeş-Bolayi Univ., Fac. of Math. Res. Sem., Seminar of Geometric Function Theory. Preprint 5, 91-98 (1986)

10.1186/1029-242X-2014-135

Cite this article as: Nunokawa et al.: Conditions for starlikeness of the Libera operator. Journal of Inequalities and Applications 2014, 2014:135

\section{Submit your manuscript to a SpringerOpen ${ }^{\circ}$ journal and benefit from:}

- Convenient online submission

- Rigorous peer review

Immediate publication on acceptance

Open access: articles freely available online

High visibility within the field

- Retaining the copyright to your article 